\title{
Making MEMS more suited for Space: Assessing the proton-radiation tolerance of structural materials for microsystems in orbit
}

\author{
Tobias Bandi ${ }^{\mathrm{a}, \mathrm{b}}$, João Polido-Gomes ${ }^{\mathrm{b}}$, Antonia Neels $\mathrm{s}^{\mathrm{a}}$, Alex Dommann ${ }^{\mathrm{a}}$ and Herbert R. Shea ${ }^{\mathrm{b}}$ \\ ${ }^{a}$ Centre Suisse d'Électronique et Microtechnique, 2002 Neuchâtel, Switzerland, \\ bÉcole Polytechnique Fédérale de Lausanne (EPFL), 2002 Neuchâtel, Switzerland
}

\begin{abstract}
We report on the susceptibility of structural MEMS materials to proton radiation damage. Radiation tests at spacerelevant doses were conducted on MEMS resonators. The two materials examined were single crystal silicon and SU-8, which are both in widespread use in microsystems. The resonance frequency was monitored for measuring minute changes of the Young's modulus. No radiation-induced changes of the elasticity were observed in the silicon devices up to fluences of $10^{13} \mathrm{~cm}^{-2}$, corresponding to a total ionizing dose (TID) of over 5.5 MRad for $10 \mathrm{MeV}$ protons. The SU-8 resonators showed a variation of less than $\pm 5.5 \%$ at doses of up to $1.4 \mathrm{Mrad}$ (TID). Chemical and structural analyses of the polymer were performed using infrared absorption spectroscopy and x-ray diffraction methods. We discuss possible mechanisms for the observed changes of the elasticity of SU-8.
\end{abstract}

Keywords: Silicon, SU-8, Microelectromechanical systems (MEMS), Radiation effects, Young's modulus, Accelerated aging, Material reliability

\section{INTRODUCTION}

Radiation-induced failure is a challenge for reliability of specific importance in avionics, space applications and nuclear reactors. The radiation tolerance of microelectromechanical systems (MEMS) is a field of increasing interest as microsystems offer distinctive advantages (low volume, power consumption and mass, batch fabrication and possible integration with control and sense electronics) and the field of applications is spreading rapidly. Failure modes related to radiation have been reviewed in [1]. The susceptibility of MEMS to radiation effects depends on the system design, the operation principle, the packaging, the shielding and the materials used. The most common failure mechanism is charge trapping in dielectrics, leading to the build-up of electrical fields, deteriorating the device performance or causing fatal failure. Although complete shielding could be envisaged, this quickly becomes prohibitive in mass and size, outweighing the advantages of miniaturization.

Designing for radiation tolerance can reduce or eliminate the susceptibility of devices to charge trapping and significantly improve their reliability [1], [2]. Other degradation mechanisms, however, can only be avoided by appropriate material selection. The electromechanical properties of materials and their influence on the device operation are related to the fabrication processes, the microstructure, the dimensions and the functional purpose. These factors determine the susceptibility to radiation induced ionization- and displacement damage effects. Ionizing damage causes the formation of excitations and electron-hole pairs leading to charge trapping or altering the chemical bonding structures. If the momentum transfer to the target atoms is sufficiently high, displacement damage occurs, leading to structural defects in the atomic lattice.

The performance of a material in microsystems may differ significantly from its properties in the bulk form or in integrated circuits. Consequently, the reliability of a material needs to be evaluated considering the system specificities. The qualification procedure must be defined accordingly. For instance, single crystal silicon is ubiquitous in integrated circuits (IC) and its radiation tolerance has been studied in detail; see [3] and references therein. Its stable crystalline structure rends silicon insensitive to ionizing damage but displacement damage introduces electrically active defects. However, in MEMS devices in which silicon is used as a structural material for moving parts, the device operation is very sensitive to the mechanical properties, such as Young's modulus, stress gradient or dislocation density. These properties are of particular interest in systems exploiting resonances for pressure-, acceleration- or rate-of-rotationsensing, actuation of devices such as micromirrors or resonators for signal processing or conditioning. The stability of vibrational modes is directly related to the Young's modulus which therefore must remain constant over time (and hence radiation dose).

Reliability, Packaging, Testing, and Characterization of MOEMS/MEMS and Nanodevices XII, edited by Rajeshuni Ramesham, Herbert R. Shea, Proc. of SPIE Vol. 8614, 86140M

(C) 2013 SPIE · CCC code: 0277-786X/13/\$18 · doi: 10.1117/12.2004705 
Surface-micromachined polysilicon resonator beams irradiated by gamma- and electron radiation have been investigated [4], [5]. A decrease of the resonance frequency and an increase of resistance were observed. The results were attributed to a combination of displacement damage, injection annealing and thermal spike effects. The effect of fast neutrons on the electromechanical properties of several materials used in microsystems has been studied and reported recently [6].

The aim of our work is to systematically investigate the effect of proton radiation on the Young's modulus of structural MEMS materials, in order to elucidate their applicability under conditions with high radiation levels. We have selected single crystal silicon and SU-8 for their importance in state-of-the-art MEMS devices. It has been previously reported by collaborators of our group that the resonance frequency of commercial silicon micromirrors had changed shortly after proton irradiation of $1 \mathrm{MeV}$ and $4.3 \mathrm{MeV}$ protons at $100 \mathrm{Mrad}$ total ionizing dose (TID) [7]. After three weeks the devices had recovered the resonance frequency prior to irradiation. This observation was explained by the introduction of defects which were mobile at room temperature and therefore annealed out after the irradiation.

Polymers are generally more sensitive to radiation than metals or semiconductors. Large changes of over $150 \%$ in the Young's modulus of PMMA have been reported after irradiation with ${ }^{60} \mathrm{CO}$ gamma-rays and protons [8]. The polymeric epoxy resin SU-8 is a strong candidate for MEMS in space. It allows the fabrication of high aspect-ratio geometries, 3D structures and bio-compatible MEMS. First investigations on its tolerance to radiation have been conducted using neutrons [9]. The hardness, i.e. the resistance to plastic deformation, has been analyzed in SU-8 coatings, and a good stability of this parameter was observed. These promising results justify more detailed investigations of the radiation tolerance of SU-8.

We have recently reported on the radiation tolerance of single crystals silicon and SU-8 microresonators in [10]. Here we present the results of further investigations made and discuss advances on possible models for understanding the radiation-induced effects. In section 2 we review the radiation environment in space and justify the selected radiation conditions. This section also contains a description of the experimental methods which were used. The results of the structural and dynamical investigations after irradiation are presented and discussed in section 3 , where we also discuss possible theoretical concepts for explaining our observations.

\section{EXPERIMENTAL}

\subsection{Radiation in space environments}

Environmental testing should reproduce the conditions encountered during operation as closely as possible. To select appropriate conditions for radiation-hardness tests in view of space missions, the in-orbit radiation environment is briefly reviewed in the next paragraph, based principally on [11] and [12].

The most abundant species in space are protons, electrons and heavy ions. The composition and intensity of radiation depend on the orbit, the space weather and the solar activity. Near Earth, charged particles trapped by the Earth's magnetic field dominate the radiation spectrum. In a spacecraft with a standard shielding thickness of 2.5-6.3 mm of aluminum (or, normalized by the material density, $0.7-1.7 \mathrm{~g} / \mathrm{cm}^{2}$ ) the dominating contribution to the absorbed dose comes from protons in the low-earth orbits (LEO) and electrons (and Bremsstrahlung) in the geostationary orbits (GEO). Proton energies lie principally in the range between $0.1 \mathrm{MeV}$ and $400 \mathrm{MeV}$. The maximum energy of electrons is $5 \mathrm{MeV}$ within distances of about 2.4 earth radii and $7 \mathrm{MeV}$ in the domains of up to 12 times the earth radius. The LEO radius is at approximately 1.1 earth radii whereas GEO corresponds to 6.7 earth radii. In the interplanetary regions, the particle spectrum is dominated by the solar activity and galactic cosmic rays. Protons are the main species of solar flares and account for $90-95 \%$ of the particles. The remaining portion of the particle emission falls to heavier ions (mostly alphaparticles). Similarly, galactic cosmic rays are mostly protons (85\%) and alpha-particles (14\%). Heavier ions contribute to less than one percent of the species. The energy of the galactic cosmic rays are significantly higher (GeV/nucleon) than for particles originating from the sun (MeV/nucleon) [11] [12]. Although heavy ions only account for a fraction of galactic cosmic rays their contribution to the total energy absorbed can be significant because the stopping power scales with $\mathrm{Z}^{2}$ [12]. This effect is illustrated in Fig. 1. Due to their high stopping power, heavy ions are an important source of single-event effects in electronic devices. However, in most MEMS components, which do not rely on thin depletion regions or p-n junctions, single-event effects are not relevant. 

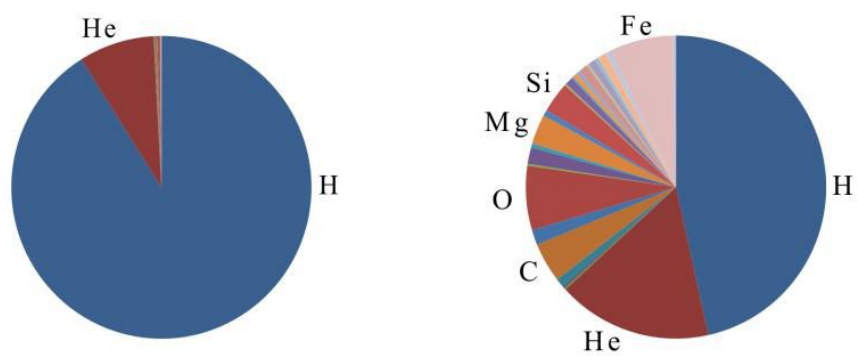

Fig. 1: (Left) Relative abundance of elements in galactic cosmic rays $\left(\mathrm{E}=1 \mathrm{GeV} /\right.$ nucleon). (Right) Relative abundance $\mathrm{x} \mathrm{Z}^{2}$ which illustrates the effect nuclear mass on the stopping power in materials. The elements are ordered after the nuclear charge Z. Adapted from [12].

This brief overview illustrates the variety of radiation types, compositions and energies which may be encountered during space missions. For testing on earth the effect of radiation is usually approximated by a limited number of radiation types and energies (e.g. conditions summarized in [13]). The total ionizing damage dose (TID) absorbed by a space system shielded by $4 \mathrm{~mm}$ aluminum is on the order of $1 \mathrm{krad} /$ year in LEO and $10 \mathrm{krad} / \mathrm{year}$ in GEO [14]. The total dose level usually requested in the qualification testing of generic EEE parts is $100 \mathrm{krad}$. For comparison, the expected ionizing dose of a tracking detector in the Large-Hadron-Collider (LHC) at CERN is $1 \mathrm{MRad}$, obtained after 1.5 years of operation, which is much higher than most space doses [15].

For our tests, we selected protons due to their importance in space environments, and their ability to penetrate shielded devices. In addition to this, protons are well suited for material screening and testing as they simultaneously cause displacement damage and ionizing damage.

\subsection{MEMS resonators for investigation of radiation-induced changes of Young's modulus}

For the purpose of isolating radiation-induced effects on the material itself we used devices which consist of only a single material. Clamped-free single crystal resonators with lengths of $1-2 \mathrm{~mm}$, width $80 \mu \mathrm{m}$, and thickness $50 \mu \mathrm{m}$ were fabricated on silicon-on-insulator (SOI) wafers by lithography and anisotropic etching. The chips were placed on PCB plates for testing and were electrostatically actuated. Polymer resonators were fabricated using a two-layer process for the chip body and the device layer respectively. The chips contained both clamped-free $(0.8 \mathrm{~mm}$ and $1 \mathrm{~mm}$ length) and clamped-clamped resonators (1 $\mathrm{mm}$ and $1.5 \mathrm{~mm}$ length) which were $120 \mu \mathrm{m}$ wide and $100 \mu \mathrm{m}$ thick. The SU-8 resonators were actuated using a piezoelectric element. The characterization of the resonances was conducted using Laser-Doppler vibrometry (LDV) under vacuum conditions. For a detailed report on the experimental setup we refer to [7]. Proton energies and doses were selected with the aim of simulating the energies and particle fluences in orbit. The total doses were calculated from the particle fluences by SRIM 2008 simulations [16]. Details on the fabrication process and the irradiation conditions were reported elsewhere [10].

The resonance frequency $f$ of the first out of plane mode of a cantilever is directly dependent on the material stiffness:

$$
f=\frac{1.875^{2}}{2 \pi} \quad \frac{t}{I^{2}} \sqrt{\frac{E}{12 \rho}}
$$

$t$ and $l$ are the thickness and length of the resonator respectively, $\rho$ is the material density and $E$ is the Young's modulus. By monitoring variations in the resonance frequency we calculated changes in the materials elasticity using the total derivative of (1). To investigate the chemical structure of the SU-8 we used infrared spectroscopy. IR-spectroscopy exploits the fact that light with specific wavelength excites characteristic vibrational and rotational modes of chemical bonds and functional groups. The absorption spectrum of a material therefore allows identifying the chemical bonds and functional groups which are present in the system. Attenuated-Total-Reflection FTIR (ATR-FTIR) vibrational spectroscopy measurements were performed using a Bruker Hyperion 3000 instrument. The infrared absorption was measured in the spectral range between $600 \mathrm{~cm}^{-1}$ and $4000 \mathrm{~cm}^{-1}$. X-ray diffraction measurements were made on a Panalytical X'Pert Pro MRD instrument. 


\section{RESULTS AND DISCUSSION}

\subsection{Single crystal silicon}

The characterization of the silicon resonators was carried out after the activity of the samples had abated to safe levels, three months after the irradiation. On each tested silicon chip at least four resonators were measured. Fig. 2 shows the combined results of the change in Young's modulus of silicon. The proton energies and fluences corresponded to total doses between $10 \mathrm{krad}$ and $5.9 \mathrm{Mrad}$.

No statistically significant change in the resonance frequency and Young's modulus were observed due to the irradiation. In some chips the standard deviation between the resonators was in the percent-range but the deviations averaged out and were not statistically significant. We note that the doses used in this study were up to 100 times higher than typical space qualification levels and that possible degradation effects would be expected to be amplified accordingly. The effect of proton irradiation on the crystal structure was also investigated by high-resolution x-ray diffraction (HRXRD) methods. We investigated the 004-Bragg peak and neither elevated diffuse scattering, peak broadening nor crystal straining were detected (see [10]). This indicates that the proton irradiation did not introduce significant amounts of stable lattice defects. The results also show that the Young's modulus of single crystal silicon was highly stable under proton irradiation.

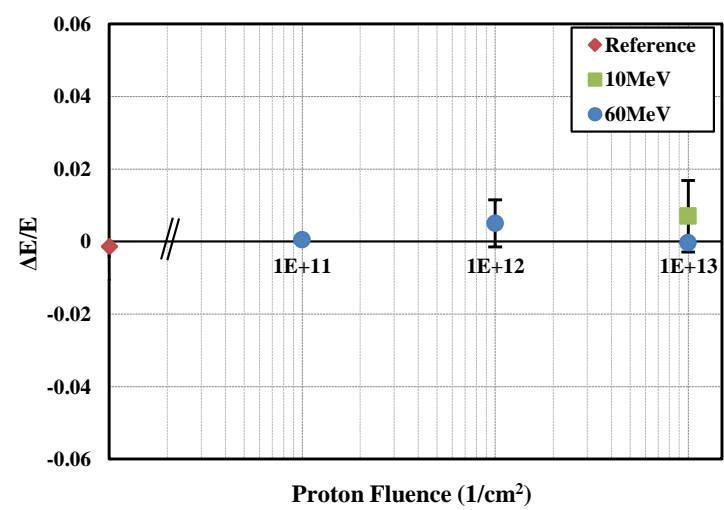

Fig. 2: Relative change in elasticity in the silicon resonators after $10 \mathrm{MeV}$ and $60 \mathrm{MeV}$ proton irradiation. Each data point represents the average and standard deviation of the results from several resonators on one chip (at least 4), irradiated under the same conditions.

\subsection{SU-8}

Proton irradiation of SU- 8 was made at energies between $10 \mathrm{MeV}$ and $200 \mathrm{MeV}$ and fluences between $10^{10} \mathrm{~cm}^{-2}$ and $3 \mathrm{x}$ $10^{12} \mathrm{~cm}^{-2}$ [10]. The total doses ranged from $0.7 \mathrm{krad}$ to $1350 \mathrm{krad}$ TID. As shown in Fig. 3 the optical appearance of the polymer remained unchanged after irradiation and no discoloration was observed.

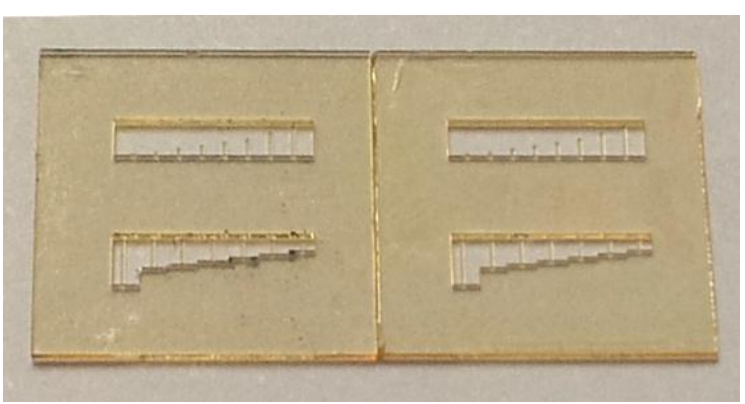

Fig. 3: Comparison between irradiated SU-8 chip and a reference sample. The left chip has been irradiated by $30 \mathrm{MeV}$ protons and a total fluence of $10^{10} \mathrm{~cm}^{-2}$. The size of the chips is $9 \mathrm{~mm} \times 9 \mathrm{~mm}$. 


\section{X-ray diffraction and IR-spectroscopy}

SU-8 was investigated by x-ray diffraction and by ATR-FTIR spectroscopy. The amorphous structure of the polymer was confirmed by x-ray diffraction, as shown in Fig. 4. A halo (near-order) peak was observed at 2-Theta angles in the range of $18-20^{\circ}$. No difference in the diffractograms was observed after irradiation. This indicates that no crystallization occurred and that the near-order structure of the polymer remained unchanged by the radiation.

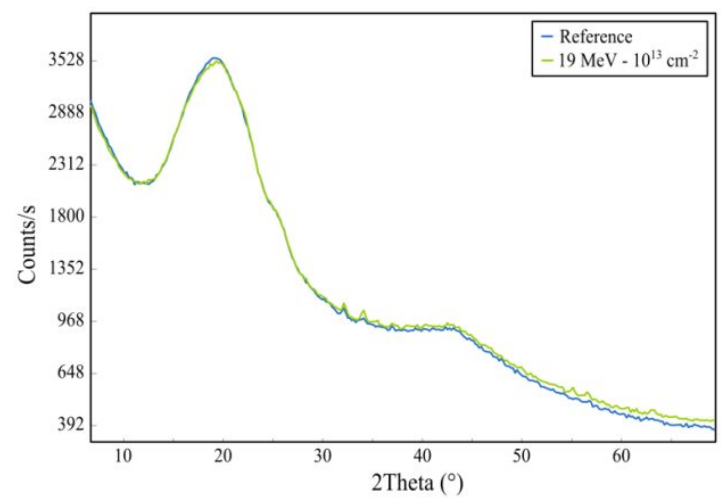

Fig. 4: X-ray diffraction pattern of SU-8. The figure shows an un-irradiated sample (blue) and a sample irradiated at $3 \times 10^{12}$ $\mathrm{cm}^{-2}$ at $19 \mathrm{MeV}$ proton energy. No change in the diffraction pattern was observed after irradiation. The faint peaks at $32^{\circ}$ and $34^{\circ}$ are artifacts from the sample holder.

It has already been reported by various authors that FTIR spectroscopy was used to support the optimization of SU-8 fabrication parameters, such as temperature, ramp rates and duration of soft bake and post-exposure bake as well as the exposure dose [17]-[19]. Also it has been shown that FTIR can support the functionalization of SU-8 surfaces with various molecules for bio-MEMS [20], [21]. Fig. 5 shows the ATR-FTIR absorption spectrum of an un-irradiated SU-8 control sample. Its peak positions and intensities were in qualitative agreement with works reported in literature and were assigned to the chemical groups according to Table 1, based on [17], [18].

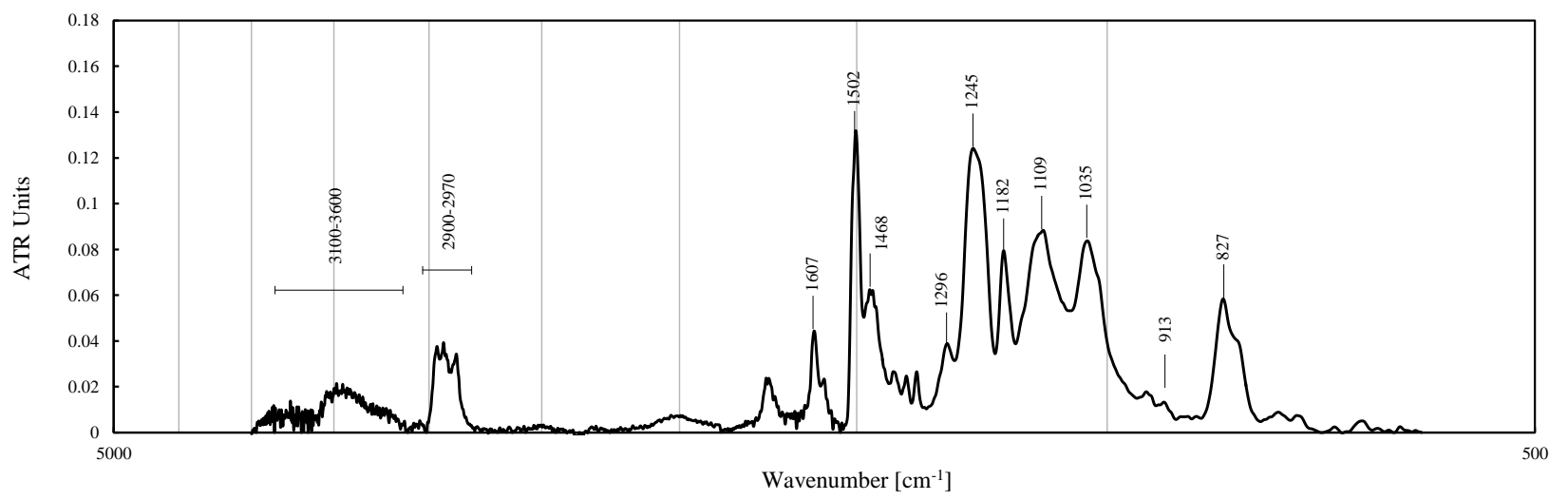

Fig. 5: Infrared absorption spectrum of an un-irradiated SU-8 sample.

The peak at $918 \mathrm{~cm}^{-1}$ which was attributed to the epoxy rings was found in the polymerized SU-8, indicating that the cured SU-8 still contained epoxy groups which had not reacted during the post-exposure bake. The other two epoxy modes were near other IR bands and were therefore not clearly discernible. The presence of epoxy groups in the cured resin is in agreement with the findings reported in [20] where it was found that the epoxy peaks did only completely disappear after an extended hard bake of 16 hours at $200{ }^{\circ} \mathrm{C}$. In [20] residual epoxy groups at the surface of the cured SU-8 were used as attachment points for functional layers. The concentration of residual epoxy groups was enough to allow the formation of coatings which enabled cell adhesion and growth. In the irradiated SU-8 specimen no additional peaks or significant changes of the IR absorption spectrum were observed. The peak at $918 \mathrm{~cm}^{-1}$ which was attributed to the epoxy rings was also present in irradiated samples and its intensity had not significantly changed. This suggests that the irradiation did not lead to heavy disintegration or modification of the polymer structure. 
Table 1: Infrared spectroscopy peak assignment for SU-8. The peaks were assigned following [17] and [18].

\begin{tabular}{ll}
\hline \hline Peak wavenumber $\left[\mathrm{cm}^{-1}\right]$ & Assignment \\
\hline $3100-3600$ & O-H stretch (water) \\
$2860-2980$ & Aliphatic C-H stretches \\
1610,1500 & Aromatic ring C-C stretch modes \\
1470 & $\mathrm{CH}_{2}$ bend (scissoring) \\
1390,1365 & Aliphatic $\mathrm{CH}_{3}$ doublet bends \\
1295,1180 & Aliphatic C-O stretches \\
$1250,915,860$ & Epoxy ring modes \\
1245 & Phenol C-O stretch \\
1110 & Aliphatic C-O stretch (ether bond) \\
1035 & Aromatic C-H in-plane bend \\
830 & Aromatic C-H out-of-plane bend \\
660 & Aromatic C-C out-of-plane bend \\
\hline \hline
\end{tabular}

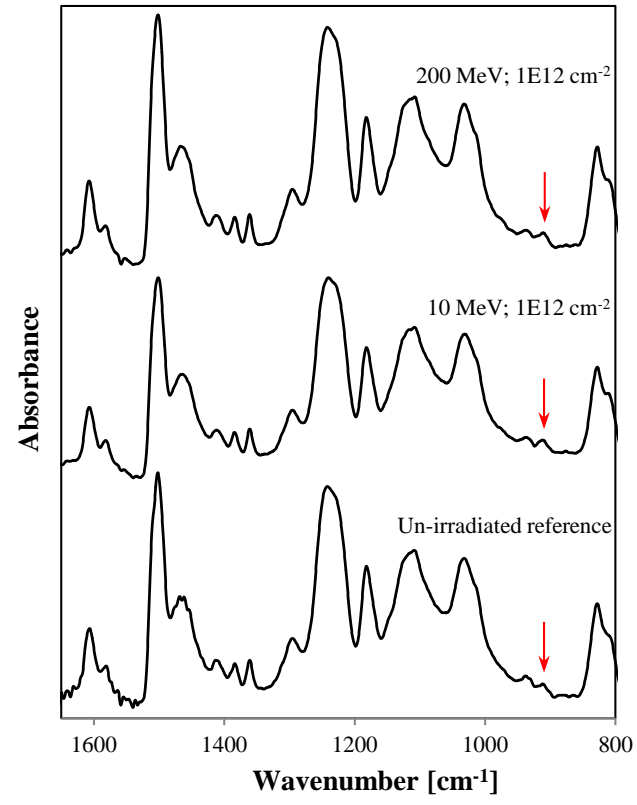

Fig. 6: Comparison of the IR spectra of an un-irradiated SU-8 chip and two irradiated specimen. The spectra are offset vertically for clarity. The red arrow shows the position of the peak at $918 \mathrm{~cm}^{-1}$ which was attributed to the epoxy rings.

\section{Resonance characteristics}

The analysis of the SU-8 resonators after irradiation showed that the Young's modulus had varied by less than $\pm 5.5 \%$. No clear dependency on the total dose and on the ion fluence was observed but the change in elasticity was correlated with the proton energy. At proton energies of $30 \mathrm{MeV}, 60 \mathrm{MeV}$, and $200 \mathrm{MeV}$ the Young's modulus of the SU-8 was reduced, while at $10 \mathrm{MeV}$ and $19 \mathrm{MeV}$ a trend to stiffening was observed. The significance was tested by a linear regression of $\Delta \mathrm{E} / \mathrm{E}$ versus the electronic stopping power and the $p$-value of both slope and intercept was below 0.01 in the measurements 2 weeks and 3 months after irradiation; therefore the null hypothesis (i.e. no correlation and no crossover between hardening and softening) was rejected. 


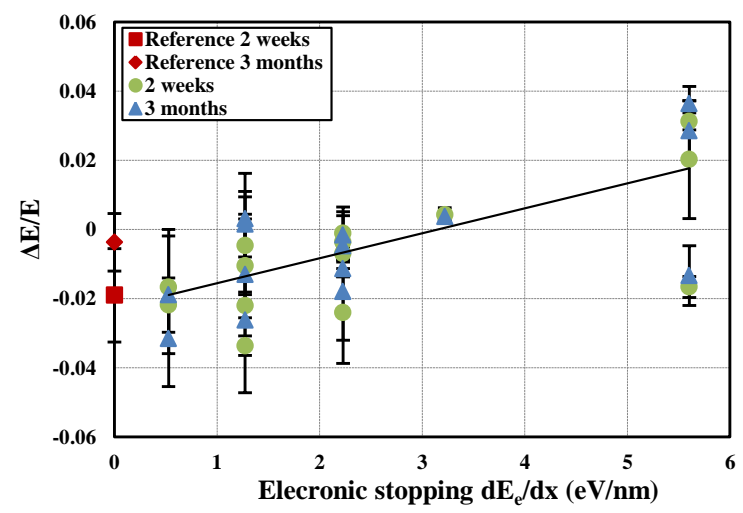

Fig. 7: Change in Young's modulus (average and standard deviation of all measured resonators on one chip) of SU-8 resonators after proton irradiation, $(\bullet) 2$ weeks after irradiation and ( $\boldsymbol{\Delta}$ ) 3 months after irradiation. The graph shows the changes versus the electronic stopping power, which is inversely proportional to the proton energy. The solid black line is a guide for the eye. Points with equal proton energy are devices irradiated at different total fluences.

\section{Reaction model for proton-induced effects on SU-8}

The particle energy is related to the stopping power $d E / d x$ by an inverse power law and thus the change in Young's modulus was also correlated to this parameter. The ion stopping power denotes the energy absorbed per unit path length in the particle track. It is closely related to the linear energy transfer (LET) which describes the energy loss from the perspective of the particle and the two terms are often used synonymously. In polymers radiation leads to ionizations, bond breaking, chain scissioning but also to the formation of new chemical bonds and polymer chain interconnects [22]. LET-dependent effects have been reported by various publications [22]-[24]. We hypothesize that the change in Young's modulus in our SU-8 resonators was caused by an LET-effect and that the stopping power caused the dominance of either cross-linking or chain scissioning. Following this line of thought, chain scissioning dominated at low LET (high proton energy) due to isolated radical formation- and displacement damage events. At high LET (lower proton energy) the energy density in the ion tracks was high enough to allow further crosslinking mediated by an elevated radical concentration. This resulted in a net hardening of the SU-8. The threshold proton energy for the crossover between softening and hardening was in the range of $20-25 \mathrm{MeV}$ which corresponds to a stopping power of approximately 3 $\mathrm{eV} / \mathrm{nm}$. This value is comparable to the findings of a previous study of poly(di- $n$-hexylsilane) where a threshold value of $10 \mathrm{eV} / \mathrm{nm}$ and a critical energy density of $\rho\left(r_{c}\right)=0.13 \mathrm{eV} / \mathrm{nm}^{3}$ was reported [23].

Although the LET is a valuable concept for quantifying the energy deposited in ion tracks, it ignores the density distribution of the absorbed energy, which determines the distribution and concentration of active species in the material [25]. A theory which allows estimating the track radii of ions has been reported in [26]. In this approach the energy distribution around the track is considered to be separated into the 'core' and the 'penumbra' regions. The radius of the core region delimits the area in which the initial electronic excitations occur. Creation of secondary electrons $(\delta$-rays) leads to a transport of energy into the penumbra region. The total average energy density $\rho(r)$ deposited within a specific radius $r$ around the ion track is [23]:

$$
\rho r=\frac{L E T}{\pi r^{2}}\left\{1-\frac{1}{2}\left[\ln \left(\frac{e^{1 / 2} r_{p}}{r_{c}}\right)\right] \ln \frac{r_{p}}{r}\right\}
$$

The core and penumbra radii are denoted by $r_{c}$ and $r_{p}$ respectively and $e$ is an exponential factor. The variables $r_{c}, r_{p}$ and $e$ depend on the particle velocity only [26]. Fig. 8 shows $\rho(r)$ for the proton irradiation at the energies used in our irradiation campaign. Assuming a single-track model the threshold proton energy of 20-25 MeV corresponds to a $\rho\left(r_{c}\right)$ of $0.09-0.12 \mathrm{eV} / \mathrm{nm}^{3}$. In case track overlapping had played a significant role, the actual energy density threshold could be higher because of the accumulation of energy and active species by several ions passing the same volume. 


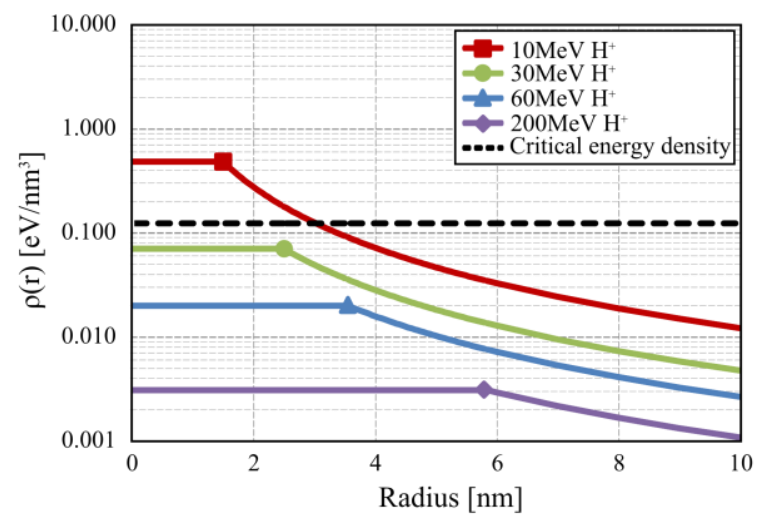

Fig. 8: Average density of energy deposition around particle track of different proton energies. The track core radius $r_{c}$ is shown as a filled circle. Inside the track core radius the energy density is homogeneous. At higher radii the energy density is determined by secondary electrons and decreases monotonically.

However, we argue that the critical energy density $\rho\left(r_{c}\right)$ obtained by the single track model explains our results best, as explained in this paragraph. A model for estimating the probability of track overlapping has been proposed in [27]. The total fractional area covered by ion tracks [25] and the probability of $n$-fold track overlapping is calculated based on the track cross-section and the total fluence. Applying this approach to our data (setting the track radius to $r_{c}$ ) indicates that at the lowest fluences $\left(10^{10} \mathrm{~cm}^{-2}\right)$ track overlapping was negligible at all proton energies. For the $10 \mathrm{MeV}$ protons a significant hardening was already observed at a fluence of $10^{11} \mathrm{~cm}^{-2}$ at which less than $3 \%$ of tracks were overlapping. At a fluence of $10^{12} \mathrm{~cm}^{-2}$ the track overlapping was over $99 \%$ for the $200 \mathrm{MeV}$ protons and $25 \%$ for the $10 \mathrm{MeV}$ protons, reflecting the different track sizes. Other publications reported the track overlapping to occur at fluences of $10^{12} \mathrm{~cm}^{-2}$ to $10^{13} \mathrm{~cm}^{-2}$, depending on the particle LET [28]-[30]. This suggests that at high proton energies and high fluences track overlapping did occur, however, the accumulated energy density was still below the threshold for the dominance of cross-linking over scissioning.

It is of value to also compare the critical energy density with the polymerization reaction of SU-8: the activation energy for the SU-8 polymerization has been reported to be $14.56 \mathrm{~kJ} / \mathrm{mol}$ [31]. This corresponds to $0.15 \mathrm{eV}$ per monomer. The density of SU-8 is $1218 \mathrm{~kg} / \mathrm{m}^{3}$ [32] (of which $10 \mathrm{wt} \%$ photoacid [33]) and the average molecular weight of one SU-8 unit is $1.4 \mathrm{ku}$ (assuming an average molecular formula of $\mathrm{C}_{88} \mathrm{O}_{16} \mathrm{H}_{100}$ ). The volume taken by a single unit of SU-8 therefore is approximately $2.2 \mathrm{~nm}^{3}$ and the threshold energy density deposited on a single SU-8 monomer is $0.15 \mathrm{eV} / 2.2 \mathrm{~nm}^{3}=0.07 \mathrm{eV} / \mathrm{nm}^{3}$. An underlying assumption is that the solvent content of the final polymer is negligible. This estimation shows that the activation energy density for polymerization is on the order of the critical energy deposition density $\rho\left(r_{c}\right)$ which was determined from the change in elasticity in the irradiated SU-8 samples as shown above. We hypothesize that the proton irradiation could have caused stiffening in the material by inducing further epoxy crosslinking reactions. Such a mechanism would require the presence of epoxy groups in the hardened resin. This is supported by the FTIR-spectroscopy measurements (Fig. 5 and Fig. 6) in which the peak assigned to the epoxy groups was present in the cured resin.

Although this scenario qualitatively fits the experimental observations and is in agreement with concepts reported in literature, several uncertainties remain. For instance, the change in Young's modulus was correlated with the proton energy but at a given energy no clear trend with the proton fluence was found (see [10]). In addition, the observed effects were small and not much larger than the experimental variance. Therefore more detailed investigations would be required to clarify the fundamental processes by which proton radiation interacts with SU-8.

\section{CONCLUSIONS}

The susceptibility to proton-irradiation damage was investigated in two types of microresonators made of singlecrystal silicon and the polymer SU-8 respectively. Whereas silicon is ubiquitous in microsystems, SU-8 is a good candidate for polymeric MEMS structures due to its excellent mechanical and chemical stability and its biocompatibility. The crystalline properties and the Young's modulus of single crystal silicon were found to be stable at proton fluences of up to $10^{13} \mathrm{~cm}^{-2}$. For $10 \mathrm{MeV}$ protons this corresponded to 5.9 Mrad TID, a dose which is much higher than typical space 
qualification levels of 20-100 krad. At proton fluences of up to $3 \times 10^{12} \mathrm{~cm}^{-2}$ the Young's modulus of SU-8 was found to vary less than $\pm 5.5 \%$. A dependency of the sign and degree of change in elasticity with the proton energy was observed and a possible model for explaining the experimental results was proposed. In this approach the dominance of either cross-linking or scissioning was related to the energy deposited in the ion tracks. Chain-scissioning dominated above the threshold energy of 20-25 MeV whereas at lower energies the stopping power was sufficiently high to establish active species concentrations which allowed further crosslinking. Although the detailed processes by which proton radiation interacts with the SU-8 are currently not fully understood we note that the resonators remained intact and fully functional after irradiation, showing that SU-8 is highly tolerant to proton radiation.

The measurement of the dynamical properties of micro-resonators is a powerful method for the investigation of material properties in structures relevant to microtechnology. Our work shows that single crystal silicon and SU-8 are tolerant to high doses of proton radiation and are well suited for MEMS in space applications. The selected geometry, fabrication process and operation mode add to the relevance and allow for transferability of these results to other types of MEMS devices.

\section{ACKNOWLEDGMENT}

We gratefully acknowledge the partial support of this work by the Networking/Partnering Initiative of the European Space Agency under Contract No. 21872. We thank H. Wojtek and Ilia Britvitch from the Paul-Scherrer institute for their support during the irradiation campaigns. We also thank T. Rohr and G. van Papendrecht from ESTEC (TEC-QTE) for very helpful discussions and for assistance in the ATR-FTIR measurements.

\section{REFERENCES}

[1] Shea H. R., "Radiation sensitivity of microelectromechanical system devices," J. Micro-Nanolith. MEM. 8(3), 031303 (2009)

[2] McClure S. S., Edmonds L. D., Mihailovich R., Johnston A. H., Alonzo P., DeNatale J., Lehman J., and Yui C., "Radiation effects in micro-electromechanical systems (MEMS): RF relays," IEEE Trans. Nucl. Sci. 49(6), 3197-3202 (2002)

[3] Srour J. R., Marshall C. J. and Marshall P. W., "Review of displacement damage effects in silicon devices," IEEE Trans. Nucl. Sci. 50(3), 653-670 (2003)

[4] Wang L., Tang J., and Huang Q.-A., "Gamma irradiation effects on surface-micromachined polysilicon resonators," J. Microelectromech. Syst. 20(5), 1071-1073 (2011)

[5] Wang L., Tang J., and Huang Q.-A., "Gamma and electron beam irradiation effects on the resistance of micromachined polycrystalline silicon beams," Sens. Actuators A Phys.177, 99-104 (2012)

[6] Gkotsis P., Kilchytska V., Fragkiadakis C., Kirby P.B., Raskin J.-P. and Francis L. A., "Effects of fast neutrons on the electromechanical properties of materials used in microsystems," J. Microelectromech. Syst. 21(6), 14711483 (2012)

[7] Gomes J. and Shea H. R., "Displacement damage effects in silicon MEMS at high proton doses," Proc. SPIE 7928, $79280 \mathrm{G}(2011)$

[8] Niklaus M., Rosset S., Shea H., "Array of lenses with individually tunable focal-length based on transparent ion-implanted EAPs," Proc. SPIE 7642, 76422K (2010)

[9] Key M. J., Cindro V. and Lozano M., "On the radiation tolerance of SU-8, a new material for gaseous microstructure radiation detector fabrication," Radiat. Phys. Chem. 71(5), 1003-1007 (2004)

[10] Bandi T., Gomes J., Neels A., Dommann A., Marchand L. and Shea, H. R., "Proton-Radiation Tolerance of Silicon and SU-8 as Structural Materials for High-Reliability Microsystems" (submitted).

[11] Stassinopoulos E. G., and Raymond, J. P., "The space radiation environment for electronics," Proc. IEEE 76(11), 1423-1442 (1988)

[12] Mewaldt R. A., "Galactic cosmic ray composition and energy spectra," Advances in Space Research 14(10), 737-747 (1994) 
[13]ESCC Basic Specification No. 22900, "Total dose steady-state irradiation test method," Issue 3 (2007) https://escies.org

[14] Hartzell A. L., daSilva M. G. and Shea H. R., [MEMS reliability (MEMS Reference Shelf Series)] Springer US, Boston MA, sec. 4.4.1. (2011)

[15] Spieler H., "Introduction to radiation-resistant semiconductor devices and circuits," AIP Conf. Proc. 7th Beam instrumentation workshop 390, 23-49 (1997)

[16]Ziegler J. F., Biersack J. P. and Littmark U., [Stopping and Range of Ions in Solids] Pergamon Press, New York, (1985)

[17] Tan T. L., Wong D., Lee P., Rawat R. S. and Patran A., "Study of a chemically amplified resist for x-ray lithography by Fourier Transform Infrared Spectroscopy," Applied Spectroscopy 58(11), 1288-1294 (2004)

[18] Keller S., Blagoi G., Lillemose M., Haefliger D. and Boisen A., "Processing of thin SU-8 films," J. Micromech. Microeng. 18(12), 125020 (2008)

[19] Wong D., Tan T. L., Lee P., Rawat R. S. and Patran A., " Study of x-ray lithographic conditions for SU-8 by Fourier transform infrared spectroscopy," Microelectronic Engineering 83(10), 1912-1917 (2006)

[20] Wang Y., Pai J.-H., Lai H.-H., Sims C. E., Backman M., Li G. P. and Albritton N. L., "Surface graft polymerization of SU-8 for bio-MEMS applications," J. Micromech. Microeng. 17(7), 1370-1380 (2007)

[21] Joshi M., Kale N., Lal R., Rao V. R. and Mukherji S., "A novel dry method for surface modification of SU-8 for immobilization of biomolecules in Bio-MEMS," Biosensors and Bioelectronics 22(11), 2429-2435 (2007)

[22] Lee E. H., "Ion-beam modification of polymeric materials - fundamental principles and applications," Nucl. Instrum. Meth. B 151, 29-41 (1999)

[23] Seki S., Maeda K., Kumini Y., Tagawa S., Yoshida Y., Kudoh H., Sugimoto M., Morita Y., Seguchi T., Iwai T., Shibata H., Asai K., and Ishigure K., "Ion beam induced crosslinking reactions in poly(di-n-hexylsilane)," $J$. Phys. Chem. B. 103(15), 3034-3048 (1999)

[24] Sasuga T., Kudoh H. and Seguchi T., "High energy ion irradiation effects on polymer materials-Changes in mechanical properties of PE, PSF and PES," Polymer 40(18), 5095-5102 (1999)

[25] Seki S., Tsukuda S., Maeda K., Matsui Y., Saeki A. and Tagawa S., "Inhomogeneous distribution of crosslinks in ion tracks in polystyrene and polysilanes," Physical Review B 70(14), 144203 (2004)

[26] Magee J. L., and Chatterjee A., "Radiation chemistry of heavy-particle tracks. 1. General considerations," $J$. Phys. Chem. 84(26), 3529-3536 (1980)

[27] Riedel C., and Spohr R., "Correcting overlapping counts in dose calibration at high event-densities," Nucl. Tracks 5(3), 265-270 (1981)

[28] Koizumi H., Ichikawa T., Yoshida H., Shibata H., Tagawa S. and Yoshida, Y., "Radical formation in the radiolysis of solid alanine by protons and helium ions," Nucl. Instrum. Meth. B 117(3), 269-274 (1996)

[29] Licciardello A. and Puglisi O., "Anomalous molecular weight distribution in ion irradiated polystyrene near the gel transition," Nucl. Instrum. Meth. B 91, 436-441 (1994)

[30] Lee E. H., Rao G. R. and Mansur L. K., "Hardness Enhancement and Crosslinking Mechanisms in Polystyrene Irradiated with High Energy Ion-Beams," Materials Science Forum 248-249, 135-146 (1997)

[31] Cho J-D., Ju H-T., Park Y-S. and Hong J-W., "Kinetics of Cationic Photopolymerizations of UV-Curable Epoxy-Based SU8-Negative Photoresists With and Without Silica Nanoparticles" Macromolecular Materials and Engineering 291(9), 1155-1163 (2006)

[32] Feng R., Farris R. J., "The characterization of thermal and elastic constants for an epoxy photoresist SU8 coating, ” J. Mater. Sci. 37(22), 4793-4799 (2002)

[33] Lorenz H., Despont M., Fahrni N., Brugger J., Vettiger P., Renaud P., "High-aspect-ratio, ultrathick, negativetone near-UV photoresist and its application for MEMS," Sens. Actuators A Phys. 64(1), 33-39 (1998)

[34]Zhang J., Tan K. L., Hong G. D., Yang L. J. and Gong H. Q., "Polymerization optimization of SU-8 photoresist and its applications in microfluidic systems and MEMS," J. Micromech. Microeng. 11(1), 20-26 (2001) 